\title{
EDITORIAL
}

\section{The parenteral opioid shortage: Causes and solutions}

\author{
Hanna Hollingsworth, PharmD; Chris Herndon, PharmD, CPE
}

Key words: Opioids, shortage, protocol, ketamine, nalbuphine, butorphanol

Growing scarcity of several parenteral opioids threatens to compromise patient care by either delaying or preventing their use in the treatment of acute pain. This shortage is a serious dilemma in healthcare, with health systems across the United States struggling to maintain dwindling supplies of morphine, hydromorphone, and fentanyl. In addition to creating barriers to timely and effective treatment, patient safety also becomes a potential concern. Reliance on higher concentration formulations of these high-alert medications increases the risk for errors leading to overdose. Although oral alternatives exist, parenteral opioids remain essential analgesics for those with swallowing or absorption limitations, post-operative pain, chest pain, and life-limiting disease.

While the problem is evident, the underlying cause of this drug shortage is less clear. The Food and Drug Administration's (FDA) drug shortage list provides ambiguous explanations such as "demand increased for drug" or even "other." 1 Predicted shortage durations vary but are likely to continue well into the next year as per manufacturer estimations. Further investigation into the parenteral opioid shortage reveals an array of underlying causes.

Every year, the Drug Enforcement Agency (DEA) sets annual Aggregate Production Quotas (APQs) for schedule I and II drugs. Creation of quotas is part of the closed system of distribution for controlled substances which aims to limit diversion and abuse. Individual quotas for each manufacturer application of a controlled substance are then set by the DEA in such a way as to not exceed the APQ for said substance. $^{2}$ The Controlled Substance Act mandates that the DEA must propose APQs and establish individual manufacturer quotas by a certain deadline each year. However, according to a Government Accountability Office (GOA) report from 2015, the DEA has a history of missing these deadlines, which in turn may play a role in causing or worsening drug shortages. ${ }^{3}$ Drug manufacturers claim that delayed quotas causes reliance on remaining inventory until the quotas are created, which prevents accumulation of reserve stock and ultimately puts them at risk for experiencing a shortage. ${ }^{3}$

Often though, the source of drug shortages originates from drug manufacturers. Considering that a substantial amount of drug shortages affects sterile injectable products, it is reasonable to question the role of quality control failure. In 2011, 56 percent of reported reasons for sterile injectable drug shortages were due to quality, but this percentage may be an underestimate. ${ }^{4}$ Although Pfizer does not state in their "Dear Customer" letter that the upgrades at their Hospira (owned by Pfizer) plant in McPherson, Kansas are a reaction to quality problems, a published warning letter from the FDA to Hospira indicates that this is precisely why its facility is undergoing upgrades. ${ }^{5}$

The warning letter details multiple violations of current good manufacturing practice regulations including, but not limited to, poor aseptic technique (specifically in relation to hydromorphone), lack of defect limits for their visual inspections, and lack of appropriate laboratory controls. ${ }^{5}$ Similar violations were discovered by the FDA at two other US based and three international Hospira plants. ${ }^{5}$ As Hospira supplies 75 percent of generic injectable opioids, its facility remediations likely made a significant impact on the current parenteral opioid shortage. ${ }^{4}$

Regardless of the cause of the opioid shortage, healthcare facilities are scrambling to find solutions. A community teaching hospital in southern Illinois implemented a pharmacist-driven intravenous opioid reduction protocol to address opioid backorders. Eligible patients with parenteral opioid orders are identified by pharmacists, who convert those orders to an oral analgesic alternative when clinically appropriate. The protocol allows for either independent dose conversions or conversion via a chart of potential oral alternatives dependent on the drug, dose, and PRN severity scale of the ordered opioid.

Utilization of ketamine and nalbuphine for analgesia were also explored as potential solutions to the parenteral opioid shortage within this institution. However, lack of nursing, physician, and pharmacy 
familiarity with these medications have proven to be a significant road block. Still, they remain viable alternatives, especially in light of limited options. A considerable amount of literature supports the use of ketamine in analgesia and has even been shown to reduce opioid consumption in patients receiving both medications. ${ }^{6}$ Nalbuphine, on the other hand, has less readily available literature describing its use in analgesia, but is nevertheless FDA approved for pain relief and has an exceptional safety profile. ${ }^{7}$

Ketamine use in analgesia has been studied extensively as bolus and infusion dosing in the emergency department and perioperative setting. Bolus dosing may be more practical when using as an analgesic and has been shown to be safe and effective in subanesthetic doses. Ketamine intravenous boluses of $0.15-0.3 \mathrm{mg} / \mathrm{kg}$ up to a maximum cumulative dose of approximately $1 \mathrm{mg} / \mathrm{kg}$ provides analgesia without significant risk of oversedation or respiratory depression. ${ }^{6,8}$ Precautions of use and contraindications should also be outlined in any protocol, with known or suspected schizophrenia (even if currently treated) being the most widely noted exclusion.?

Nalbuphine and butorphanol are mixed agonistantagonist parenteral opioids which serve as analgesic options for acute pain treatment in patients not already receiving opioid agonists. ${ }^{10}$ Given their mixed mechanism of action, $\mu$-opioid dependent patients would be at high risk of withdrawal with nalbuphine or butorphanol use, thus precluding their use in this patient population. A cursory review of the relative equianalgesic potency of nalbuphine and butorphanol is warranted, however the actual conversion from a mu-opioid agonist to one of these agents is not recommended. Nalbuphine is equipotent to parenteral morphine (1:1) while butorphanol is approximately five times more potent (1:5).10 Nalbuphine and butorphanol share a favorable side effect profile, with a ceiling effect observed in regard to respiratory depression. ${ }^{7}$ Even so, patients receiving either of these agents should still receive the same monitoring of vitals and sedation that is used with other opioids. Both agents may produce psychotomimetic side effects largely owing to their agonist effects at the kappa opioid receptor.

Unfortunately, drug shortages, especially of sterile injectable products, will likely continue. Even with increased and more timely reporting of actual and potential drug shortages with the enactment of the Food and Drug Safety and Innovation Act in 2011, these shortages can still result in significant hardships for healthcare facilities and patients alike. Potential solutions are complex but likely involve creating manufacturer incentives for quality products. ${ }^{4}$ As health care providers, we must act to promptly create countermeasures that protect the provision of patient care. A blend of creativity, safeguards, and identifying as many interprofessional advocates as possible are fundamental parts to creating a successful policy, procedure, or protocol in response to drug shortages.

Hanna Hollingsworth, PharmD, PGY-1 Pharmacy Resident, HSHS St. Elizabeth's Hospital, St. Louis, Missouri.

Chris Herndon, PharmD, CPE, Professor, Southern Illinois University-Edwardsville School of Pharmacy, Edwardsville, Illinois.

\section{REFERENCES}

1. FDA: FDA Drug Shortages. Available at https://www.access data.fda.gov/scripts/drugshortages/. Accessed February 7, 2018.

2. Section 826 of Title 21 United States Code Controlled Substances Act. Available at: https://www.deadiversion.usdoj.gov/ 21cfr/21usc/826.htm. Accessed February 7, 2018.

3. Office USGA. GAO-15-202, DRUG SHORTAGES: Better Management of the Quota Process for Controlled Substances Needed; Coordination between DEA and FDA Should Be Improved. 2015. Available at https://www.gao.gov/assets/ 670/668252.pdf. Accessed February 7, 2018.

4. Woodcock J, Wosinska M: Economic and technological drivers of generic sterile injectable drug shortages. Clin Pharmacol Ther. 2013; 93(2): 170-176. doi:10.1038/clpt.2012.220

5. FDA: 2017-Hospira Inc 2/14/17. Available at: bttps:// www.fda.gov/iceci/enforcementactions/warningletters/2017/ ucm 542587.htm. Accessed February 7, 2018.

6. Galinski M, Dolveck F, Combes X, et al.: Management of severe acute pain in emergency settings: Ketamine reduces morphine consumption. Am J Emerg Med. 2007; 25(4): 385-390. doi:10.1016/j.ajem.2006.11.016

7. Barash PG: Clinical Anesthesia. Available at: https://books.google.com/books?id=exygUxEuxnICEdq=Clinical + Anesthesia,$+7 e+$ barash + butorphanolEsource $=$ gbs_navlinks_s. Accessed February 7, 2018.

8. Beaudoin FL, Lin C, Guan W, et al.: Low-dose Ketamine Improves Pain Relief in Patients Receiving Intravenous Opioids for Acute Pain in the Emergency Department: Results of a Randomized, Double-blind, Clinical Trial. Acad Emerg Med. 2014; 21(11): 1194-1202. doi:10.1111/acem.12510

9. Green SM, Roback MG, Kennedy RM, et al.: Clinical practice guideline for emergency department ketamine dissociative sedation: 2011 update. Ann Emerg Med. 2011; 57(5): 449-461. doi:10.1016/j.annemergmed.2010.11.030

10. Pharmacist's Letter: Equianalgesic Dosing of Opioids for Pain Management. Available at: http://pharmacistsletter.ther apeuticresearch.com/pl/ArticleDD.aspx? nidchk $=1 \xi c s=\varepsilon s=P L E p$ $t=2 \xi s e g m e n t=4662 \xi d d=280821$. Published 2015. Accessed February 7, 2018. 